\title{
Rigorous evaluation of the edge-focusing wiggler based on the magnetic field measurement
}

\author{
Shigeru Kashiwagi, ${ }^{*}$ Ryukou Kato, Akihito Mihara, Takanori Noda, and Goro Isoyama \\ Institute of Scientific and Industrial Research (ISIR), Osaka University, 8-1 Mihogaoka, Ibaraki, Osaka 567-0047, Japan \\ Kimichika Tsuchiya, Tatsuro Shioya, and Shigeru Yamamoto \\ Institute of Materials Structure Science, High Energy Accelerator Research Organization (KEK), 1-1 Oho, Tsukuba, \\ Ibaraki 305-0801, Japan
}

(Received 9 February 2009; published 30 December 2009)

\begin{abstract}
The edge-focusing (EF) wiggler, which produces the strong field gradient for transverse focusing incorporated with the normal wiggler field, has been fabricated to evaluate its performance rigorously with the magnetic field measurement. It is a five-period planar wiggler with an edge angle of $2^{\circ}$ and a period length of $60 \mathrm{~mm}$. The magnetic field in the wiggler is measured using Hall probes at four different wiggler gaps. It is experimentally confirmed that a high field gradient of $1.0 \mathrm{~T} / \mathrm{m}$ is realized, as designed, along the beam axis in the EF wiggler. The magnetic field gradient of the EF wiggler is derived as a function of the magnetic gap. The field gradient decreases with increasing magnet gap more slowly than the peak magnetic field does for the present experimental model. An analytic formula for the field gradient of the EF wiggler is derived and it is shown that the slope of the field gradient with the magnet gap can be changed by varying the magnet width of the EF wiggler.
\end{abstract}

DOI: 10.1103/PhysRevSTAB.12.120703

PACS numbers: 41.60.Cr, 41.85.Lc, 07.55.-w

\section{INTRODUCTION}

Since the free-electron laser (FEL) was proposed and experimentally demonstrated in the late 1970s, extensive research and development have been conducted to realize its potential advantages over conventional lasers, such as higher power, higher efficiency, wider wavelength tunability, and a wide range of operational wavelengths $[1,2]$. These features are being realized, through not necessarily simultaneously, and one of the solutions for such problems is self-amplified spontaneous emission abbreviated as SASE $[3,4]$, which can be operated in principle at any wavelengths, because it does not use mirrors for an optical resonator limiting the operation wavelength. SASE has attracted attention as the fourth generation synchrotron light source producing high-intensity coherent $\mathrm{x}$ rays and not only extensive studies have been conducted but also user facilities are being constructed [5-7].

SASE is a single-pass FEL without seed light. When a high energy and high brightness electron beam enters a long undulator, or sometimes called a wiggler, synchrotron radiation emitted at the entrance by the electron beam works as a seed and it is amplified exponentially as the electron beam moves in the wiggler. The wiggler has to be long enough to amplify coherent light to a power level reaching saturation; it is as long as $100 \mathrm{~m}$ in the $\mathrm{x}$-ray SASE projects under construction [8,9]. The spatial density of electrons in the beam has to be high to make the gain length shorter, which is defined as the wiggler length

\footnotetext{
*Corresponding author. shigeruk@sanken.osaka-u.ac.jp.
}

necessary for amplifying the SASE power by a factor equal to the base of the natural logarithm $e$ [4], so that the electron beam has to be focused to keep the electron beam size small all through the long wiggler.

A conventional planar wiggler of the horizontal oscillation type focuses an electron beam appreciably in the vertical direction, which is called the natural focusing, only when its energy is sufficiently lower than $100 \mathrm{MeV}$ and the wiggler field is strong or more precisely the K-value is large, but does not focus it in the horizontal direction in any case. If such a beam is injected to the wiggler with the matching condition in the vertical direction, the vertical beam size will remain constant due to the natural focusing as it moves along the wiggler. In the horizontal direction, however, the beam size will increase and diverge unless the external focusing force is provided.

In the early 1990s, a few schemes for transverse focusing in the wiggler were proposed to enhance the gain of conventional FELs [10-12], one of which presents the idea of the edge-focusing (EF) wiggler [11]. Based on the idea, a wiggler incorporated with the transverse field gradient was fabricated but the evaluation of its performance was not quantitative [13]. Subsequently since the late 1990s, various schemes have been proposed or investigated to provide the focusing force in both directions in the wiggler for SASE in the short wavelength range so that the beam size is kept small over the whole length of the very long wiggler [14-18]. In these schemes, either pole faces are shaped or permanent magnet blocks are added in the wiggler gap, in order to produce the field gradient for focusing the beam. The magnet gap of the wiggler is sacrificed in some of these methods or the good field region 
is limited in the other methods. To meet stringent requirements to the wiggler for SASE, we revaluated the idea of the EF wiggler and analyzed its performance with numerical calculation [19]. The edge-focusing (EF) wiggler made only of permanent magnet blocks can produce the high magnetic field gradient superimposed on the normal wiggler field. The EF wiggler has an advantage that the strong field gradient can be produced with no obstacles in the wiggler gap and the good field region is as large as that of the normal wiggler field. As a next step of our study, we have fabricated an experimental model of the EF wiggler. In the fabrication process of this model wiggler, we developed a new method to make a low-error wiggler without adjustment of the magnetic field by trial and error [20]. The wiggler parameters of the experimental model have been chosen to meet requirements from our SASE experiment in the far-infrared region being conducted at the Institute of Scientific and Industrial Research (ISIR), Osaka University $[21,22]$. In this paper, we will report results of performance evaluation of the EF wiggler based on the magnetic field measurement.

\section{EDGE-FOCUSING WIGGLER}

The EF wiggler is basically a Halbach-type wiggler made only of permanent magnet blocks, but their shapes are not rectangular parallelepipeds. A schematic drawing of the EF wiggler for one period is shown in Fig. 1. The shape of the magnet blocks with vertical magnetization is an isosceles-trapezoidal right prism with an edge angle $\phi$, while that with longitudinal magnetization is a parallelepiped with the same angle. The average focusing force of the EF wiggler can be calculated using transfer matrices with a fringing field for a simple model consisting of a series of alternating bending magnets with the edge angle and a drift space in between [23]. The average horizontal and the vertical focusing forces, $k_{x}$ and $k_{y}$, in the EF wiggler of the horizontal oscillation type are, respectively, given by

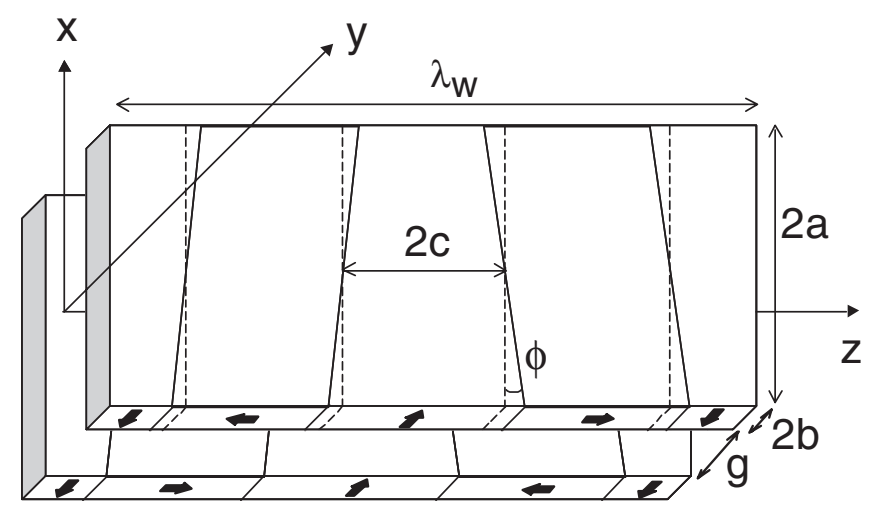

FIG. 1. Schematic drawing of a period of the edge-focusing (EF) wiggler with the edge angle $\phi$.

$$
\begin{gathered}
k_{x}=\frac{1}{B \rho}\left(\frac{\partial B_{y}}{\partial x}\right)=\frac{4 e}{m_{0} c} \frac{B_{0}}{\gamma} \times \frac{\phi}{\lambda_{w}}, \\
k_{y}=k_{0}-k_{x}=\frac{8-\pi}{3 \pi}\left(\frac{e}{m_{0} c}\right)^{2}\left(\frac{B_{0}}{\gamma}\right)^{2}-k_{x},
\end{gathered}
$$

where $B_{0}$ is the peak magnetic field, $\lambda_{w}$ the period length, and $\partial B_{y} / \partial x$ the horizontal gradient of the vertical component of the magnetic field of the EF wiggler. $B \rho$ is the magnetic rigidity and $\gamma$ the Lorentz factor of the electron. $e$ is elementary charge, $m_{0}$ the rest mass of electron, $c$ the speed of light, and $k_{0}$ the natural focusing force of the wiggler. From Eq. (1), the field gradient is calculated as

$$
\frac{\partial B_{y}}{\partial x}=4 \frac{B_{0}}{\lambda_{w}} \phi .
$$

It is found in this study that Eq. (3) is not an appropriate formula for calculating the field gradient in the EF wiggler, and this problem will be discussed in Sec. V C. Anyway, the field gradient is proportional to the edge angle and, hence, the focusing force along the wiggler can be easily adjusted with the edge angle $\phi$. Note that the sum of the vertical and the horizontal focusing forces of the EF wiggler is equal to the natural focusing force, that is, $k_{0}=$ $k_{x}+k_{y}$. A planer wiggler has an edge angle of $\phi=0$, so that no horizontal focusing force works. When the horizontal focusing force produced by an EF wiggler lies between zero and the natural focusing force $k_{0}$, both $k_{x}$ and $k_{y}$ are positive, so that the electron beam is focused simultaneously in the horizontal and the vertical directions, which is referred to as the weak focusing. For a higher energy electron beam or a lower magnetic field, $k_{x}$ surpasses $k_{0}$, so that $k_{y}$ becomes negative and accordingly the electron beam is defocused in the vertical direction. In such a case, a strong focusing scheme may be applied for double focusing using the EF wiggler with edge angles of alternating polarities.

Figure 2 shows a two-dimensional distribution of the vertical component of the magnetic field $B_{y}$ (hereafter referred to as the vertical magnetic field) on the median plane of an EF wiggler with the edge angle $\phi=5^{\circ}$, the period length $\lambda_{W}=60 \mathrm{~mm}$, the magnet gap $30 \mathrm{~mm}$, the magnet block width $100 \mathrm{~mm}$, the block height $20 \mathrm{~mm}$, and the residual induction of permanent magnet $B_{r}=1.32 \mathrm{~T}$, which is calculated with a three-dimensional magnetic field calculation program (3D program) based on the magnetic charge method [24]. The vertical magnetic field varies with the horizontal position linearly almost over the wiggler width, irrespective of the longitudinal position. Thus the field gradient producing horizontal beam focusing is nearly constant along the wiggler axis.

\section{EXPERIMENTAL MODEL}

We have applied the weak focusing scheme for the experimental model of the EF wiggler, so that all the 


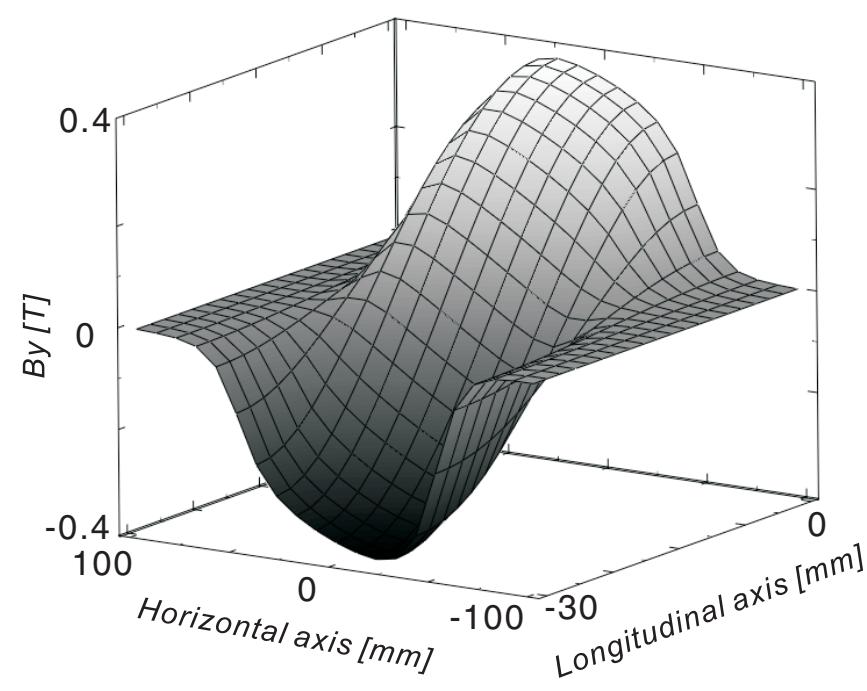

FIG. 2. Vertical magnetic field $B_{y}$ of the EF wiggler with an edge angle of $5^{\circ}$ calculated for a half wiggler period. The period length is $60 \mathrm{~mm}$ and maximum field strength on the beam axis is $0.37 \mathrm{~T}$ at the magnet gap of $30 \mathrm{~mm}$. A steep negative slope may be seen along the horizontal axis irrespective of the longitudinal position.

magnet blocks have the same edge angle. It is a five-period wiggler with the period length $\lambda_{W}=60 \mathrm{~mm}$ and the edge angle $\phi=2^{\circ}$. The main parameters of the EF wiggler are listed in Table I. The permanent magnet is Nd-Fe-B with the residual induction of $1.32 \mathrm{~T}$, which is the average value of measured residual induction for all the magnet blocks used in the EF wiggler, and standard dimensions of the magnet blocks are $2 a \times 2 b \times 2 c=(100 \times 20 \times$ 15) $\mathrm{mm}^{3}$. Figure 3 shows one of the magnet arrays of the experimental model of the EF wiggler. The lines may be seen in centers of the magnet blocks. These magnet blocks are made of two magnet pieces glued together so that angular errors of magnetization are canceled out in each magnet block. A preliminary result of this study is reported in Ref. [20]. Each magnet block is not only glued onto a

TABLE I. Main parameters of the experimental model of the EF wiggler.

\begin{tabular}{ll}
\hline \hline Period length & $60 \mathrm{~mm}$ \\
Number of periods & Five periods \\
Magnet gap & $30-60 \mathrm{~mm}$ \\
Calculated peak magnetic & $B_{0}=0.432 \mathrm{~T}$ \\
$\quad$ field at gap $=30 \mathrm{~mm}$ & \\
$\quad$ with the 3D program & \\
Edge angle & $\phi=2.0^{\circ}$ \\
Averaged field gradient & $\partial B_{y} / \partial x=1.0 \mathrm{~T} / \mathrm{m}$ \\
$\quad\left(\phi=2^{\circ}\right)$ at & \\
$\quad$ gap $=30$ mm & $\mathrm{Nd}-\mathrm{Fe}-\mathrm{B}$ \\
Permanent magnet & $B_{r}=1.32 \mathrm{~T}$ \\
Residual induction of magnet & $(100 \times 20 \times 15) \mathrm{mm}^{3}$ \\
Nominal block size $(2 a \times 2 b \times 2 c)$ & \\
\hline \hline
\end{tabular}

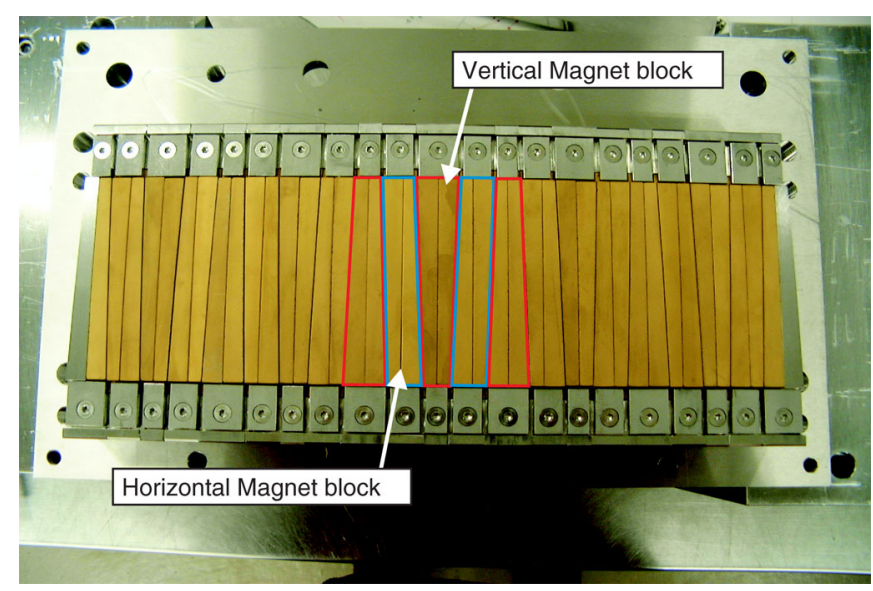

FIG. 3. (Color) Lower magnet array of the experimental model of the EF wiggler. The magnet blocks with vertical magnetization have a trapezoidal shape (rimmed with the red lines), while those with longitudinal magnetization have a parallelogram shape (with the blue lines).

holder but also mechanically clamped at both sides of each block. The arrangement of the magnet blocks was determined based on measured values of the surface magnetic field for all the magnet blocks so that effects of strength errors of magnetization on the electron orbit are minimized. The magnetic gap of the wiggler is mechanically fixed, but it can be varied, though stepwise, by changing planks connecting the upper and the lower magnet arrays.

The peak magnetic field of the wiggler at a magnet gap $g=30 \mathrm{~mm}$ is calculated using the $3 \mathrm{D}$ program to be $0.43 \mathrm{~T}$. The field gradient of the model wiggler averaged over a wiggler period is approximately $1 \mathrm{~T} / \mathrm{m}$ at $30 \mathrm{~mm}$ gap and the edge angle of $2^{\circ}$. This edge angle is chosen so that a $11.5 \mathrm{MeV}$ electron beam is focused equally in the horizontal and the vertical directions in the EF wiggler at the gap $30 \mathrm{~mm}$, which is a typical condition for our SASE experiments in the far-infrared region.

\section{MAGNETIC FIELD MEASUREMENT}

In order to evaluate performance of the model of the EF wiggler, the magnetic field has been measured at the High Energy Accelerator Research Organization (KEK), using a magnetic field measurement system with two Hall probes for simultaneous measurement of the vertical and the horizontal magnetic fields. The Hall probes for the vertical and the horizontal field measurements are separately mounted on two small copper plates, the temperature of which is controlled within a variation of $\pm 0.01^{\circ} \mathrm{C}$ to reduce spurious drift of the measured magnetic field owing to its temperature variation. They were calibrated against a NMR sensor, so that the magnetic field can be measured with an accuracy of $1 \times 10^{-5} \mathrm{~T}$. Figure 4 shows the experimental setup for the magnetic field measurement of the EF wiggler. Each Hall probe is attached at the tip of an arm equipped with two tilt stages for adjusting the orientation 

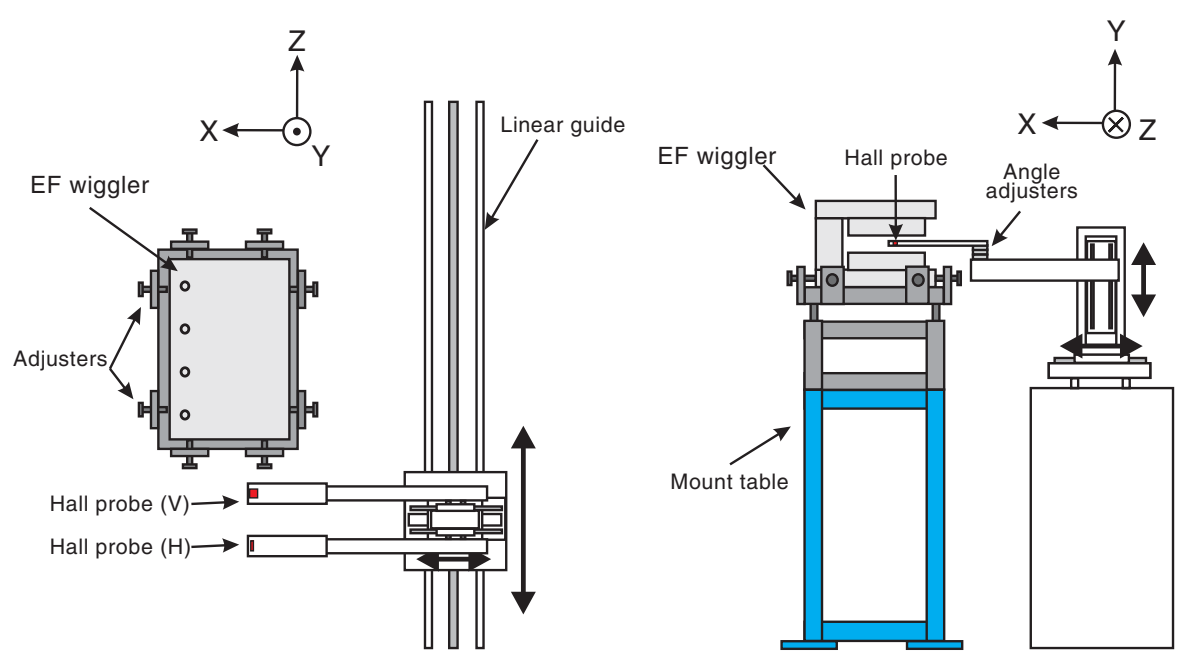

FIG. 4. (Color) Schematic drawing of the magnetic field measurement system for the EF wiggler. Left is a top view and right is a side view. Each Hall probe is installed at the tip of an arm equipped with two tilt stages and a horizontal rotation stage for adjusting the orientation of the Hall probe. The two arms with the Hall probes are mounted on a 3-axis linear stage with two vertical linear stages for adjustment of relative heights.

of the Hall probe, and a rotation stage for adjusting the horizontal direction of the arm. The two arms are mounted on a 3-axis linear stage with two vertical linear stages for independent adjustment of their heights and the magnetic field is measured simultaneously in the horizontal and the vertical directions along the wiggler axis, which is referred to as the $z$ axis, at various transverse positions, designated as the $x$ axis in the horizontal direction and as the $y$ axis in the vertical direction so that the field mapping in the wiggler is made. The origin of the coordinate system is set at the mechanical center of the wiggler magnet system, which is the middle point in the longitudinal and the horizontal directions on the median plane. When the magnetic field measurement system is first set up, the $z$ axis of the EF wiggler is set parallel to the longitudinal axis or the $z$ axis of the 3-axis linear stage with an angular accuracy of $50 \mu \mathrm{rad}$. The transverse positions of the two Hall probes are adjusted and made equal with respect to the EF wiggler and to each other. A measured value of the horizontal magnetic field is most affected by the orientation error of the Hall probe introduced by a tilt around the $z$ axis, because the vertical component of the magnetic field is much larger than the other components on and near the median plane of the wiggler. The orientation of the Hall probe for the horizontal direction is, therefore, adjusted using the two tilting stages so that intrusions of the vertical field component are minimized by measuring the horizontal magnetic field at the centers of the vertically and the longitudinally magnetized blocks, where the absolute value of the vertical magnetic field becomes maximum and zero, respectively. The orientation of the Hall probe for the vertical direction is not critical because the vertical magnetic field is the largest. It is adjusted so that absolute value of the measured magnetic field becomes maximum at centers of the vertically magnetized blocks.
In spite of these careful adjustments of the measurement system, it turns out that the remaining small orientation errors of the Hall probes and of the 3-axis linear stage for them with respect to the model wiggler lead to large errors in the magnetic field and the field gradient because the magnetic field varies appreciably to all the three directions, so that the measured magnetic field has to be corrected for the measurement errors. A detailed description of error sources and corrections against them is given in the Appendix.

\section{RESULTS AND DISCUSSIONS}

\section{A. Wiggler field and electron trajectory}

Figure 5 shows magnetic fields of the $\mathrm{EF}$ wiggler at a magnet gap of $30 \mathrm{~mm}$ measured along the beam axis or the $z$ axis. The solid circles in panel (a) show the vertical magnetic field $B_{y}$ measured along the central axis at $x=$ $y=0 \mathrm{~mm}$ and the solid circles as well as the dotted line in panel (b) show horizontal magnetic fields $B_{x}$ measured at three different transverse positions of $x=0$ and $y=0$, $\pm 2 \mathrm{~mm}$. The horizontal magnetic field $B_{x}$ on the median plane should be zero due to the geometrical symmetry, but $B_{x}$ measured on the median plane at $y=0$ varies with the longitudinal position, which is shown by the dotted line in panel (b), owing probably to imperfections of the permanent magnet blocks, though the range of the variation is less than $\pm 1 \mathrm{mT}$. The same variation remains in the magnetic field distributions measured at other vertical positions, so that the magnetic field distributions at $y=$ $\pm 2 \mathrm{~mm}$ shown in panel (b) are derived by subtracting the measured magnetic field at $y=0$. Then the measured horizontal magnetic fields shown in panel (b) are corrected for an orientation error of the Hall probe estimated to be $\theta_{x}=0.7 \mathrm{mrad}$ and $\phi_{x}=\pi / 2$ with the method given in 

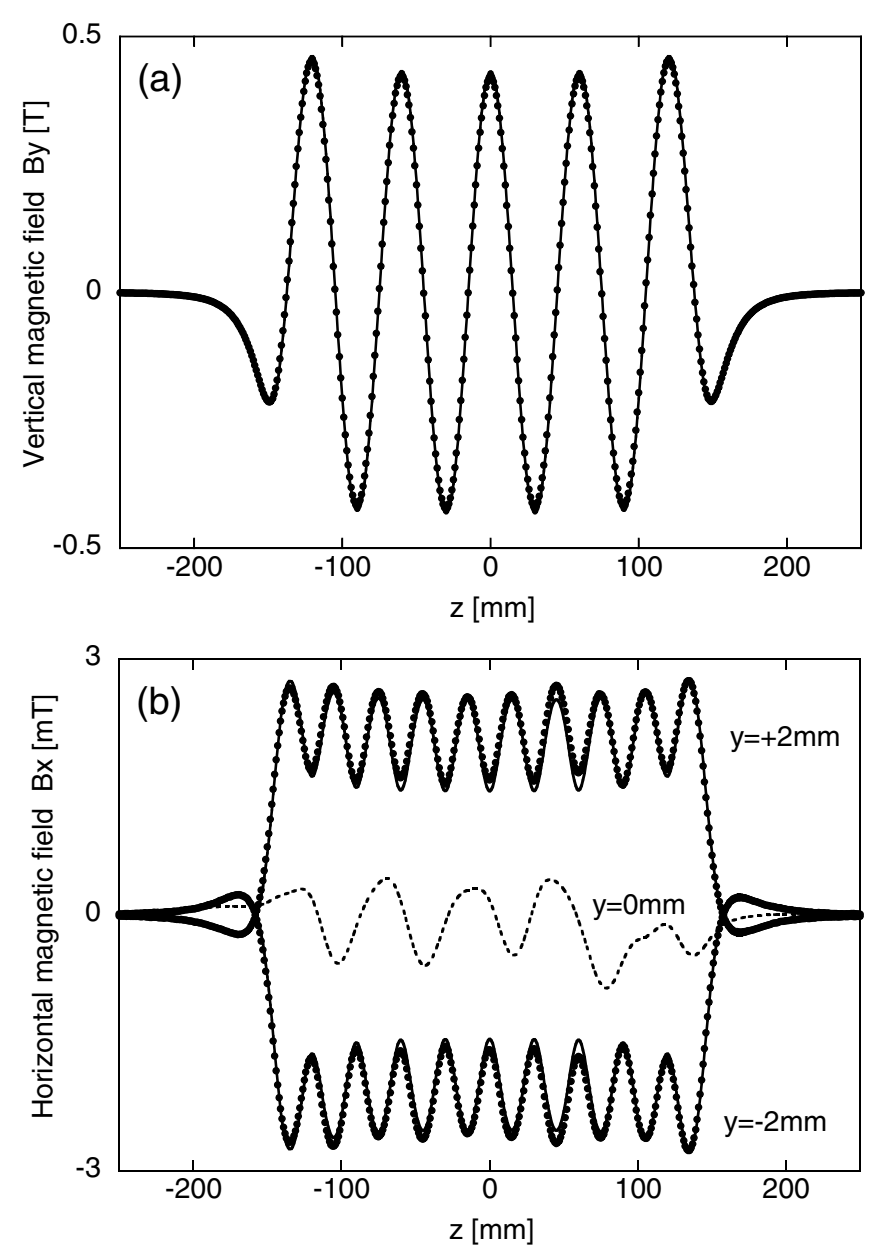

FIG. 5. Magnetic field of the EF wiggler at a magnet gap of $30 \mathrm{~mm}$ measured along the beam axis ( $z$ axis): (a) the vertical magnetic field and (b) the horizontal magnetic field. The solid circles and the dotted line show measured values and the solid lines show calculated ones. The vertical magnetic field is measured along the central axis of the wiggler at $x=y=0 \mathrm{~mm}$, while the horizontal magnetic field is measured at three different transverse positions of $x=0$ and $y=0, \pm 2 \mathrm{~mm}$. The magnetic field at $y=0$ shown with the dotted line in panel (b) is the measured values, while the magnetic fields at $y= \pm 2 \mathrm{~mm}$ are derived from the measured values at $y= \pm 2 \mathrm{~mm}$ by subtracting the magnetic field at $y=0$.

the Appendix. Since the number of periods of the model wiggler is only five, the end effect shows up in the distribution of $B_{y}$ shown in panel (a), that the peak magnetic field becomes higher toward both ends except for the last negative peaks, where half size magnet blocks with vertical magnetization are used to make the transverse position and the angle of the outgoing electron beam the same as those of the incoming electron beam.

The magnetic field distribution of the EF wiggler is numerically calculated with the $3 \mathrm{D}$ program using the parameters listed in Table I and shown by the solid lines in Fig. 5 for comparison with the measured values. The calculated magnetic field distributions agree quite well with the measured ones. The peak magnetic field at the center of the wiggler $(x=y=z=0 \mathrm{~mm})$ calculated with the $3 \mathrm{D}$ program is $B_{0}=0.432 \mathrm{~T}$, which agrees fairly well with a measured value of $B_{y}=0.421 \mathrm{~T}$.

As can be seen in Fig. 5, the magnetic field of the model wiggler shows appreciable end effects because the number of periods is small. In order to evaluate the performance of a long wiggler, we propose a new method to make use of a pseudowiggler made imaginarily from the measured magnetic field of a short model wiggler. Here we assume a pseudowiggler of 35 periods, the magnetic field of which is calculated by adding seven sets of the measured magnetic field of the model wiggler after longitudinally and progressively shifting them by five periods or $300 \mathrm{~mm}$ $(=60 \mathrm{~mm} /$ period $\times 5$ periods $)$ with respect to every contiguous unit. The total length of the pseudowiggler is $2.1 \mathrm{~m}$, which is similar to our $1.92 \mathrm{~m}$ long, 32 period wiggler with the same period length of $60 \mathrm{~mm}$ used for our FEL and SASE experiments. Figure 6 shows the vertical magnetic field of the pseudowiggler at $g=30 \mathrm{~mm}$ and the calculated electron trajectory in it. In the pseudowiggler, two pairs of longitudinal magnetization blocks of appropriate strength are added at both ends of the wiggler, as commonly adopted recently, so that the wiggler axis moves back to the line extended by the incoming and outgoing electron beam and does not move when the magnet gap is varied. The magnetic field distribution shown in Fig. 6(a) seems uniformly periodic. Indeed, the standard deviation of the peak magnetic field for the inner 31 periods excluding two periods at both ends is only $0.22 \%$. In the calculation of the second field integral in the pseudowiggler, which is proportional to an electron trajectory, a virtual end

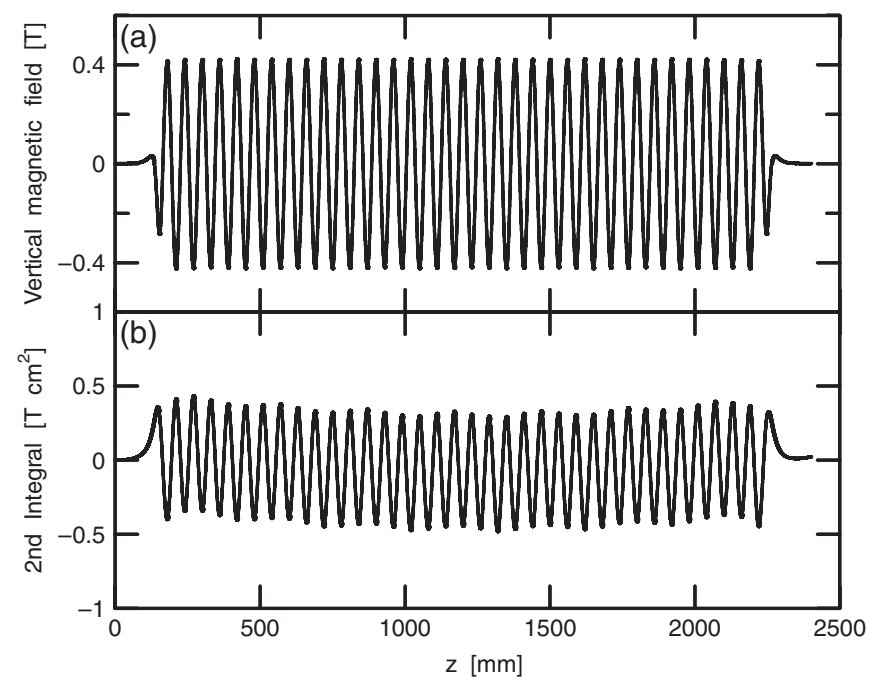

FIG. 6. Vertical magnetic field distribution and its second integral of a 35 period pseudowiggler consisting of seven sets of the measured magnetic field distribution for the model wiggler at the gap of $30 \mathrm{~mm}$ : (a) the vertical magnetic field and (b) its second integral, which is proportional to the electron trajectory. 
correction of $2.9 \times 10^{-5} \mathrm{~T} \mathrm{~m}$, which is half the integrated magnetic field of the model wiggler, is added at both ends of each model wiggler so that the field integral becomes equal to zero. This artificial correction is necessary in this model because a small field integral inevitably remaining due to the end effect produces a large second integral at the end of the pseudowiggler, which is $1.8 \mathrm{~m}$ away from the end of the farthest model wiggler, and contributions by the seven model wigglers add up coherently. The second integral of the pseudowiggler shown in Fig. 6(b) is close to the ideal electron trajectory in the wiggler, though a periodic distortion due to small magnetic field errors of the model wiggler shows up slightly. As a conclusion, the EF wiggler works as a wiggler and its magnetic field is as good as that of a normal wiggler.

\section{B. Field gradient}

The field gradient of the model wiggler is derived from the magnetic field measured at several transverse positions along the longitudinal position ( $z$ axis). Figure 7 shows the vertical magnetic field on the median plane $B_{y}(x, y=$ $0, z=0$ ) for the magnet gap $g=30 \mathrm{~mm}$ as a function of the horizontal position ( $x$ axis) measured at the middle of the model wiggler $z=0$, where the vertical magnetization block is located. The vertical magnetic field varies linearly with the horizontal position over the wiggler width and the field gradient is derived from the slope of a straight line fitted to the measured values as shown in Fig. 7. The electron beam receives the strong focusing force in the horizontal direction produced by this field gradient.

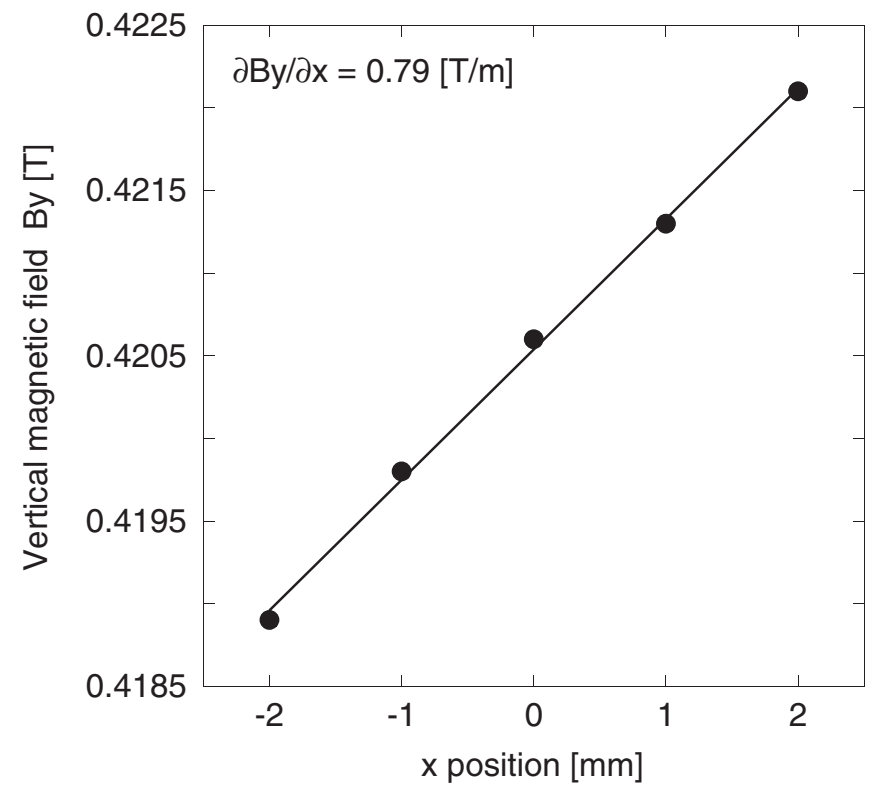

FIG. 7. Measured magnetic field $B_{y}(x, y=0, z=0)$ as a function of the horizontal position $x$ at the middle of the wiggler $z=$ 0 (on vertically magnetized blocks). The solid circles are measured magnetic fields and the line is a fit of a straight line to them for deriving the field gradient.
Figure 8 shows field gradients in the horizontal and the vertical directions $\partial B_{y} / \partial x$ and $\partial B_{x} / \partial y$ along the central wiggler axis on the median plane as a function of the longitudinal position $z$. The solid circles in Fig. 8 show the field gradient derived from the measured magnetic field. In deriving the field gradients, the measured magnetic field is corrected for measurement errors due to orientation errors of the Hall probes and of the 3-axis linear stage using the method given in the Appendix before calculating the field gradients. The orientation error of the Hall probe is estimated to be $\theta_{x}=0.7 \mathrm{mrad}$ and $\phi_{x}=\pi / 2$, and that of the linear stage to be $\psi_{x}=0.9 \mathrm{mrad}$ and $\zeta_{x}=\pi / 2$. The solid lines in Fig. 8 show transverse field gradients calculated for the model wiggler with the 3D program. The calculated field gradients agree well with the measured ones. The field gradients oscillate 10 times high and low along the wiggler axis of the model wiggler, or twice in a wiggler period. The mechanical structure and magnet properties of the EF wiggler have reflection symmetry with respect to the transverse plane at the center of the magnet arrays at $z=0$, which is a minimum position of
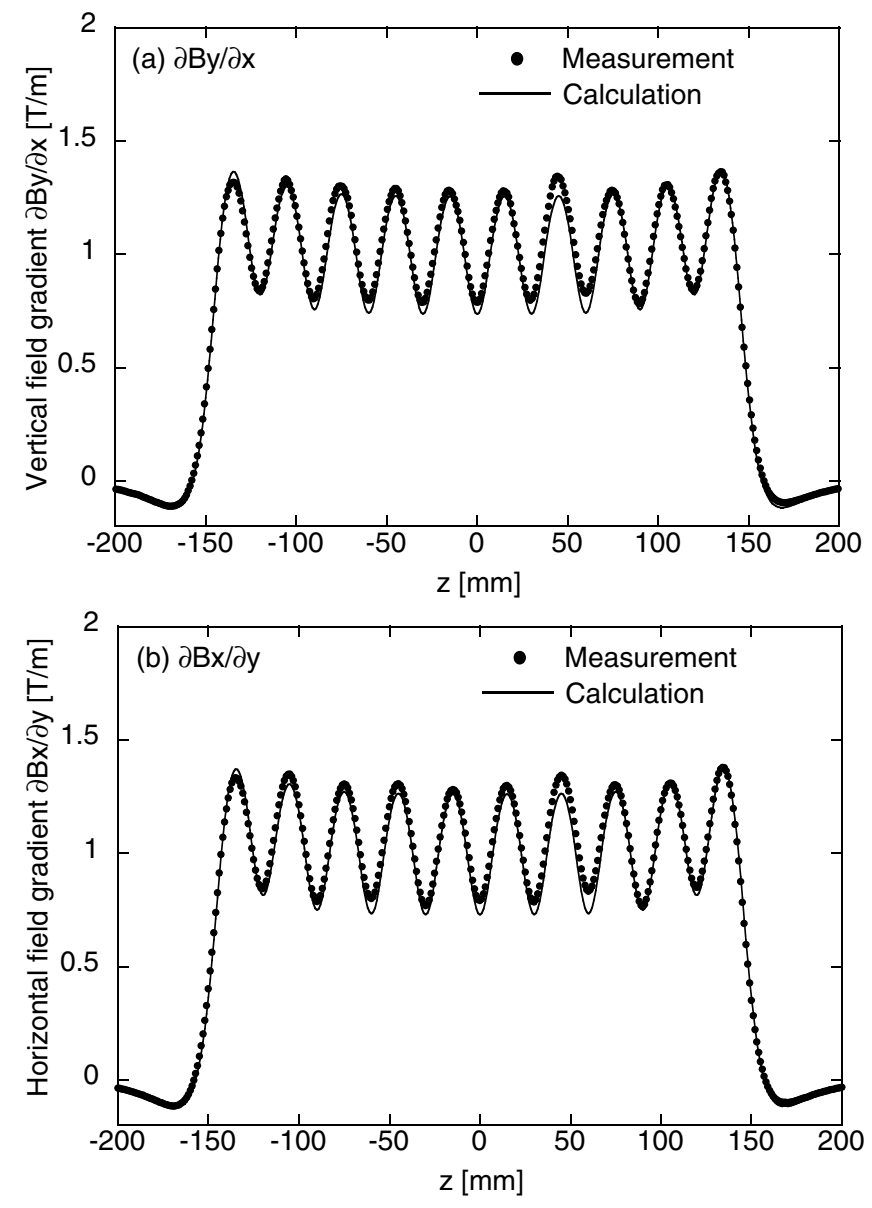

FIG. 8. Field gradients of the EF wiggler along the wiggler axis: (a) vertical field gradient $\left(\partial B_{y} / \partial x\right)$ and (b) horizontal one $\left(\partial B_{x} / \partial y\right)$. The solid circles and the solid lines show the measured field gradients and the calculated ones, respectively. 
absolute value of the field gradient shown in Fig. 8, and hence the field gradient should also be symmetric to it. The horizontal and the vertical field gradients of the model wiggler should be equal to each other according to Maxwell's equations, and they are so at a glance in Fig. 8. Actually, the measured field gradient in the horizontal direction integrated along the longitudinal axis is only $0.3 \%$ smaller than that in the vertical direction. In comparison between integrals of the measured and the calculated field gradients, however, the measured values are larger than the calculated ones by $3.2 \%$ in the horizontal direction and by $3.4 \%$ in the vertical direction. The reason for this systematic difference in the magnitudes of the measured and calculated field gradients is not clear. Possible sources of this discrepancy may be systematic error of the edge angle in the fabrication of magnet blocks and the permeability of the permanent magnet that is assumed to be 1.0 in the $3 \mathrm{D}$ magnetic field calculation
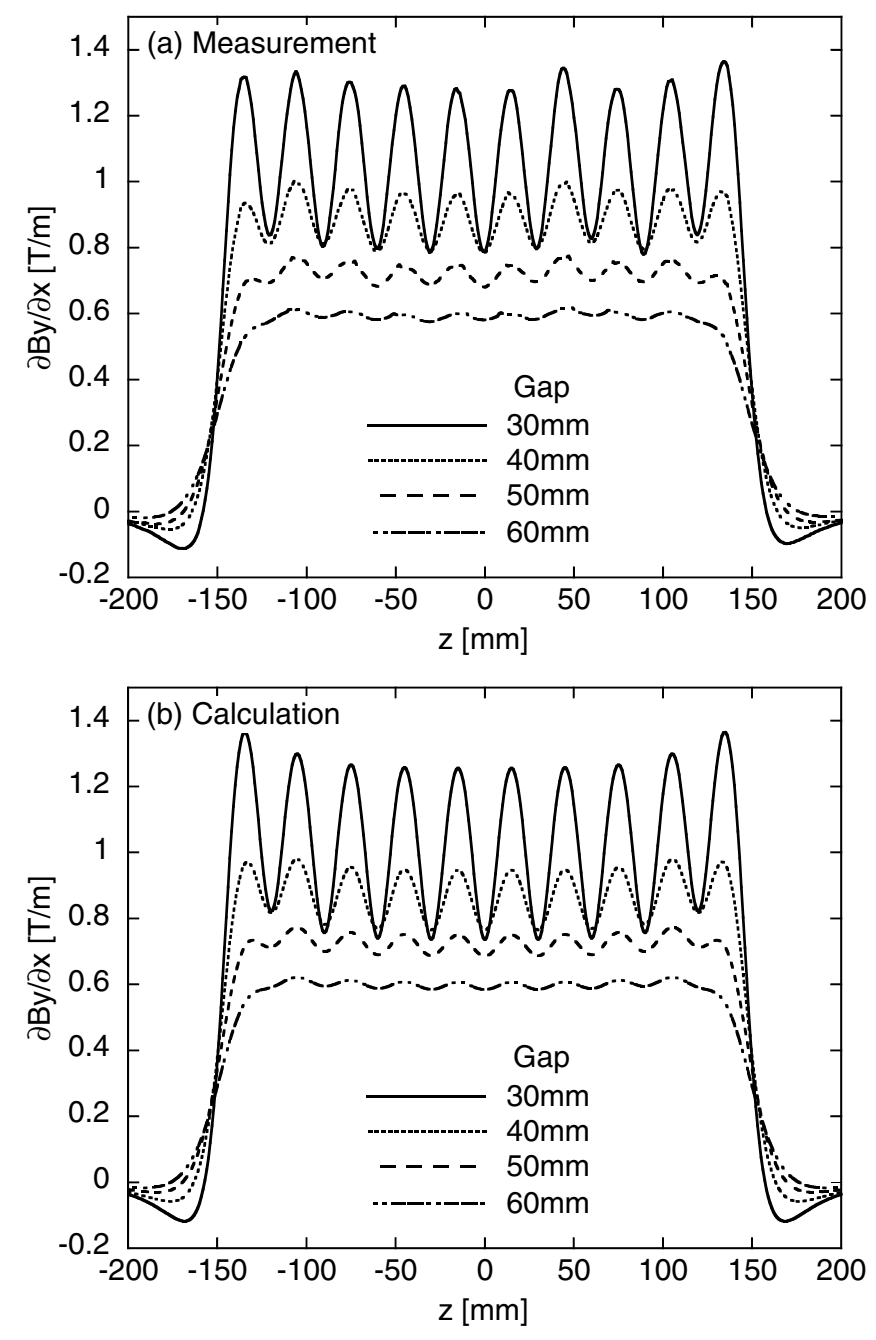

FIG. 9. Measured and calculated vertical field gradients $\left(\partial B_{y} / \partial x\right)$ of the EF wiggler along the wiggler axis at wiggler gaps of $30,40,50$, and $60 \mathrm{~mm}$. though the actual value is 1.05 in the magnetization axis and it is 1.0 in the other axes [25].

We varied the magnet gap of the EF wiggler by changing planks connecting the upper and the lower bases for the magnet arrays and measured the magnetic field at gaps $g=$ $30,40,50$, and $60 \mathrm{~mm}$. Figure 9 shows measured vertical field gradients $\partial B_{y} / \partial x$ (a) and calculated ones (b) along the axis of the model wiggler at the four wiggler gaps. The agreement is good between measurement and calculation with the 3D program in the amplitude and the oscillation period. The field gradient averaged over one wiggler period is shown in Fig. 10, together with the peak magnetic field, as a function of the magnet gap. The solid circles and the open circles show the measured peak magnetic field and the averaged field gradient of the EF wiggler, respectively. The solid and the dashed lines show the peak magnetic field and the averaged field gradient calculated with the 3D program, respectively. The measured values are in good agreement with the numerical calculation as can be seen in Fig. 10. The peak magnetic field of the wiggler decreases exponentially with increasing wiggler gap [26] and the averaged field gradient also decreases exponentially, but the slope of the field gradient is gentler than that of the peak magnetic field. The peak field at the gap $60 \mathrm{~mm}$ decreases to one-fifth of the value at $30 \mathrm{~mm}$, whereas the field gradient at $60 \mathrm{~mm}$ decreases to a half of the value at $30 \mathrm{~mm}$ only. This behavior is favorable in the view of the beam dynamics in the wiggler. The simple model based on transfer matrices fails to explain the gap dependency, because the field gradient of the EF wiggler given by Eq. (3) is proportional to the peak magnetic field. The

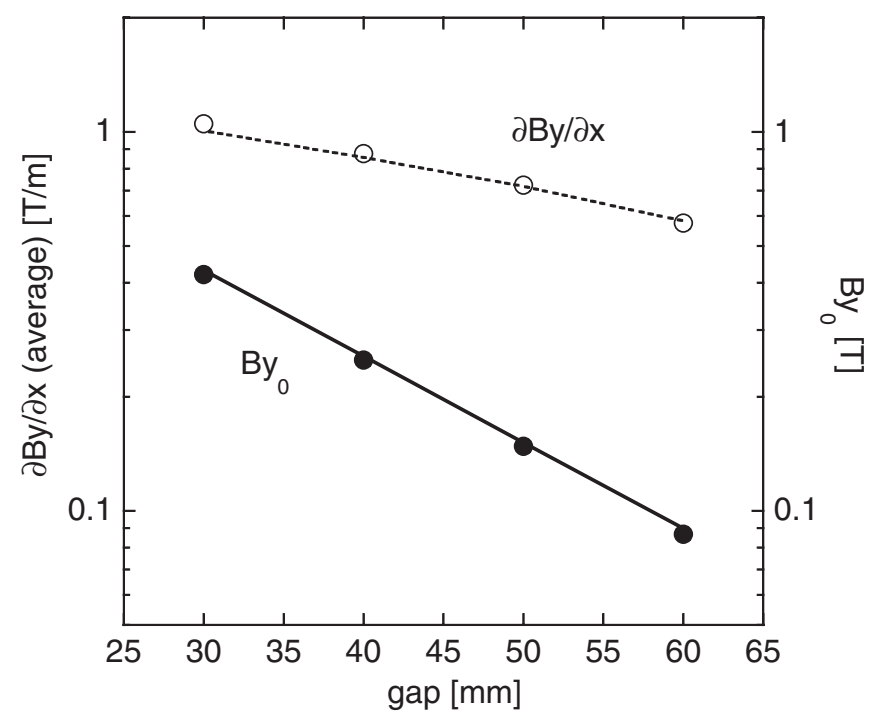

FIG. 10. Peak magnetic field and the field gradient of the EF wiggler as a function of the wiggler gap. The solid circles and the open circles show the measured peak magnetic field and the average field gradient over a wiggler period, respectively. The solid and the dashed lines show calculation using the 3D program. 
magnet gap dependency of the field gradient of the EF wiggler may be, however, understood as follows.

\section{Formula for field gradient}

The magnet structure of the EF wiggler may be expressed as four rows of triangular prisms of permanent magnet blocks in the $z$ direction superimposed to that of a normal planar wiggler as shown schematically in Fig. 11(a). The quadrupole field for beam focusing is produced by these magnet blocks and may be derived from the magnetic field in the EF wiggler calculated with the $3 \mathrm{D}$ program by subtracting the normal wiggler field. Figure 11(b) shows a result of such calculation for $B_{y}$ in the present model wiggler with the magnet gap of $30 \mathrm{~mm}$. The transverse gradient $\partial B_{y} / \partial x$ is clearly seen though it varies

(a)

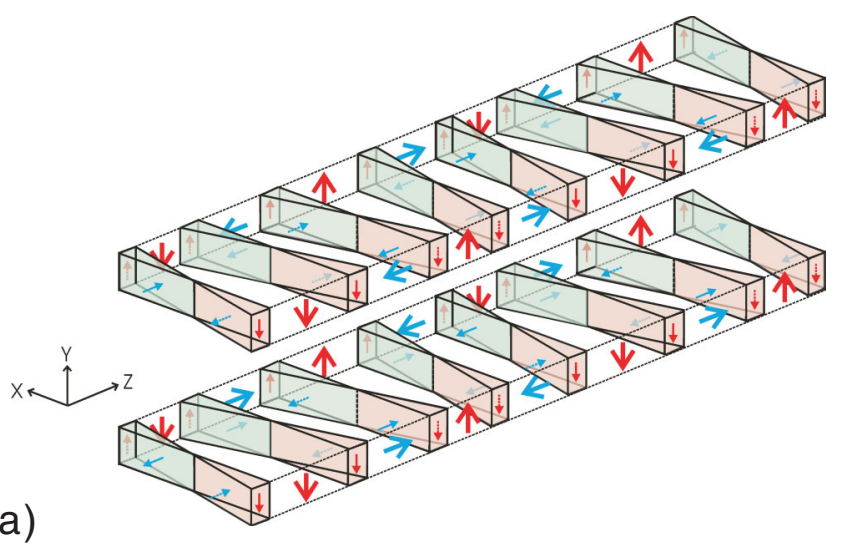

(b)

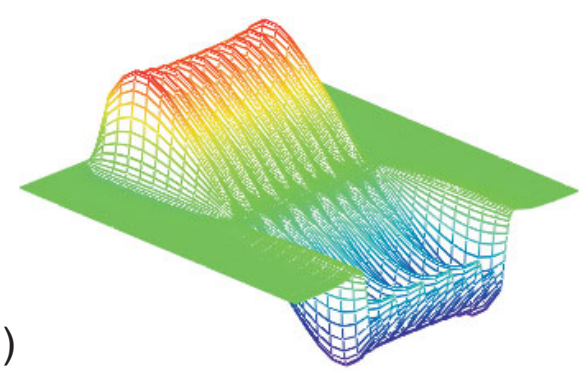

FIG. 11. (Color) Magnet structure and the magnetic field dedicated to producing the field gradient in the experimental model of the EF wiggler. (a) Magnet structure producing the field gradient. The EF wiggler consists of four rows of triangular prisms in the $z$ direction superimposed to the magnet structure of a conventional planar wiggler. Although each prism has magnetization in the $y$ direction and the $z$ direction, the $y$ magnetization mainly contributes to the field gradient. All the prisms in the upper and lower rows in the positive $x$ side have the downward magnetization and those in the negative $x$ side have upward magnetization. (b) The magnetic field $B_{y}$ on the median plane produced only by the four rows of the triangular prisms. The magnetic field is derived from that of the EF wiggler calculated with the $3 \mathrm{D}$ program by subtracting the magnetic field of the conventional planar wiggler. The magnetic field distribution shows a one-cycle oscillation in the $x$ direction with a linear field gradient near the origin. periodically in the $z$ direction as can be seen also in Figs. 8 and 9. The magnetic field distribution in the $x$ direction shows one-cycle oscillation and seems similar to that of the normal wiggler with a single period if the relatively small periodical variation in the $z$ direction is neglected. The magnetization of the triangular shaped prisms shown in Fig. 11 is twofold; one is the longitudinal magnetization in the $z$ direction and the other is the vertical one in the $y$ direction. Because the edge angle is small and, hence, the length along the $z$ direction is short, the magnetomotive force in the $z$ magnetization is small compared with that in the $y$ magnetization. Furthermore, directions of the $z$ magnetization are opposite to each other in the adjacent prisms in the same row in the $z$ direction, so that the integrated value of the magnetic field in the median plane produced by the $z$ magnetization may be negligibly small due to cancellation. The magnetic field shown in Fig. 11(b) is produced mainly by the $y$ magnetization of the triangular prisms. The triangular prisms in the upper and the lower rows in the $z$ direction have the same direction of magnetization on one side and those on the other side have the opposite direction. Based on the magnetic field distribution and the magnetic structure shown in Fig. 11, the magnetic field $B_{y}(x)$ produced by the triangular prisms may be approximated with a normal wiggler with a single period for the $x$ direction and may be given [26] as

$$
\begin{aligned}
B_{y}(x)= & 2 B_{r m} \cdot \exp \left(-\pi g / \lambda_{T}\right) \cdot\left(\frac{\sin (\pi / M)}{\pi / M}\right) \\
& \cdot\left\{1-\exp \left(-2 \pi h / \lambda_{T}\right)\right\} \cdot \sin \left(\frac{2 \pi}{\lambda_{T}} \cdot x\right),
\end{aligned}
$$

where $B_{r m}$ is an effective residual induction of the magnet, $g$ the magnet gap, $\lambda_{T}$ the effective period length of the magnetic field in the $x$ direction, $M$ the number of different directions of magnetization in a wiggler period, and $h$ the block height. The transverse field gradient is calculated to be

$$
\begin{aligned}
\frac{\partial B_{y}(x)}{\partial x}= & \frac{4 \pi}{\lambda_{T}} B_{r m} \cdot \exp \left(-\pi g / \lambda_{T}\right) \cdot\left(\frac{\sin (\pi / M)}{\pi / M}\right) \\
& \cdot\left\{1-\exp \left(-2 \pi h / \lambda_{T}\right)\right\} \cdot \cos \left(\frac{2 \pi}{\lambda_{T}} \cdot x\right) \\
\approx & \frac{4 \pi}{1.8} \cdot \frac{B_{r} \cdot \tan \phi}{\lambda_{W}} \cdot \exp \left(-\pi g / \lambda_{T}\right) \cdot\left(\frac{\sin (\pi / 2)}{\pi / 2}\right) \\
& \cdot\left\{1-\exp \left(-2 \pi h / \lambda_{T}\right)\right\} \cdot \cos \left(\frac{2 \pi}{\lambda_{T}} \cdot x\right) .
\end{aligned}
$$

The effective residual induction may be calculated as the mean magnetization of a half of the magnet blocks in the $x$ direction to be $B_{r m}=B_{r}(a \cdot \tan \phi / 4 c)=B_{r}(2 a$. $\left.\tan \phi / \lambda_{W}\right)$ using the sizes of the magnet block $(2 a \times 2 b \times$ $2 c)$, the period length of wiggler $\left(\lambda_{W}\right)$, the edge angle $(\phi)$, and the residual induction of magnet $\left(B_{r}\right)$. As can be seen in Fig. 11, the triangular prisms have two opposite direc- 


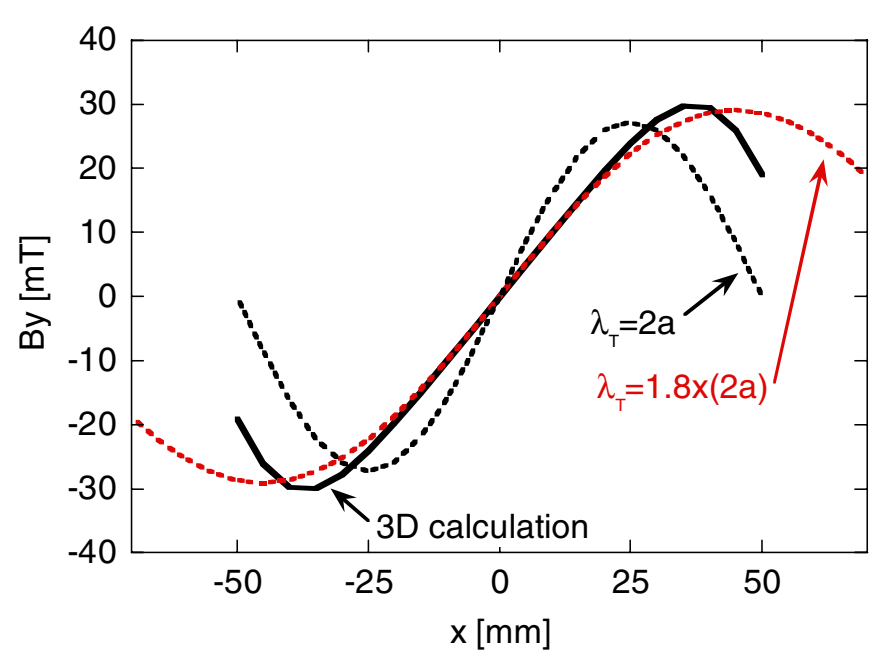

FIG. 12. (Color) Magnetic field distributions of the EF wiggler dedicated to beam focusing. The black solid line shows the magnetic field calculated with the 3D program, the black dotted line shows that calculated with Eq. (4) for $\lambda_{T}=2 a$, the horizontal width of the EF wiggler, and the red dotted line shows that calculated with $\lambda_{T}=1.8 \times(2 a)$.

tions of magnetization so that we may set $M=2$. The block height is given by $h=2 b$. Figure 12 shows magnetic fields calculated with Eq. (4) and with the 3D program as a function of the horizontal position $x$. The black solid line shows the magnetic field calculated with the 3D program and averaged in the central one period of the EF wiggler. Its distribution shows a quasisinusoidal shape but it is appreciably distorted so that the linear dependence on the $x$ direction is extended. The black dotted line shows the magnetic field distribution calculated with Eq. (4) for $\lambda_{T}=$ $2 a$, which is the magnet width of the EF wiggler. Its field gradient near the origin is higher than that calculated with the 3D program and Eqs. (4) and (5) fail to reproduce the field gradient. This is because the magnetization of the triangular prisms integrated in the $z$ direction increases linearly with the distance from the origin in the $x$ direction while the uniform magnetization in the magnet block is assumed for the effective residual induction $B_{r m}$ in Eqs. (4) and (5). This effect of nonuniform magnetization may be taken into account by using an effective period length in the $x$ direction. The magnetic field distribution denoted by $\lambda_{T}=1.8 \times(2 a)$ and plotted with the red dotted line is a result of the fit reproducing the field gradient near the origin, which means that the transverse period should be 1.8 times longer than the magnet width $2 a$. It is confirmed with the 3D calculation that the scale factor 1.8 is applicable over the wide range of magnet sizes of the EF wiggler. Figure 13 shows the field gradient as a function of the wiggler gap for EF wigglers with the same edge angle $2^{\circ}$ but with different wiggler periods and magnet widths. The circles and the squares indicate field gradients calculated with the 3D program and averaged over a wiggler period. The solid and the dashed lines show field

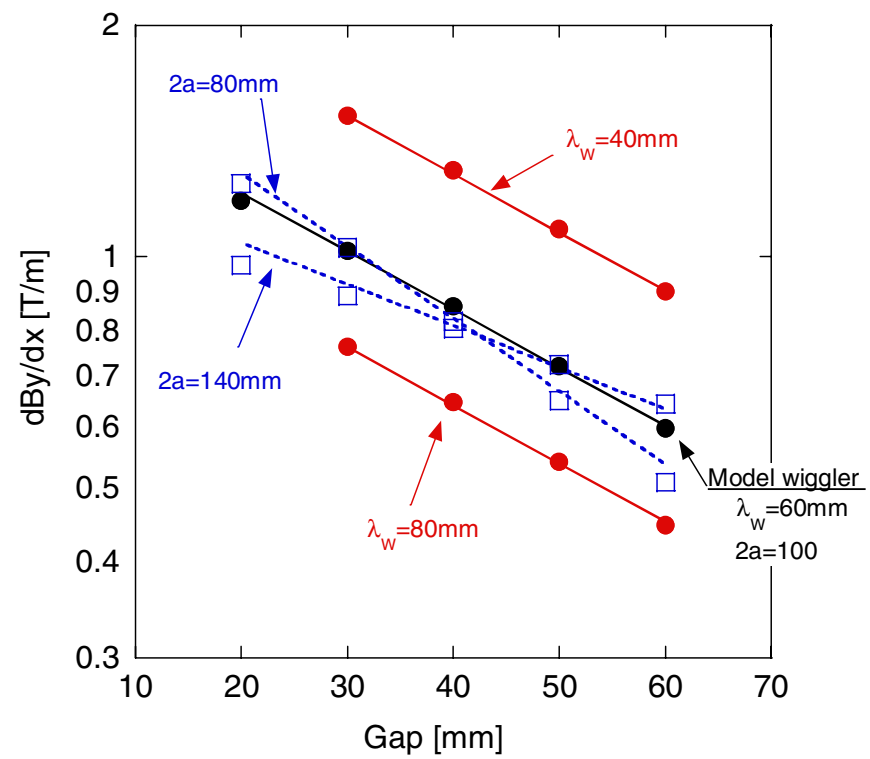

FIG. 13. (Color) Vertical field gradient as a function of the wiggler gap for EF wigglers with the same edge angle $2^{\circ}$ but with different wiggler periods and magnet widths. The circles and the squares indicate field gradients calculated with the $3 \mathrm{D}$ program and averaged over a wiggler period. The solid and the dashed lines show field gradients calculated with Eq. (5). See text for details.

gradients calculated with Eq. (5). These field gradients are calculated for three different wiggler periods of 40 , 60 , and $80 \mathrm{~mm}$ and for three different magnet widths of 80 , 100, and $140 \mathrm{~mm}$, yet results of calculation with Eq. (5) agree quite well with those with the $3 \mathrm{D}$ program. The following two points should be noted; the slope of the field gradient does not change with the wiggler period provided that the magnet width is kept constant and the slope can be varied for the constant wiggler period by changing the magnet width of the EF wiggler, as can be clearly seen in Eq. (5). Thus, the field gradient and its magnet gap dependence of the EF wiggler are quantitatively explained with Eq. (5) and it can be used for design of the EF wiggler.

Although the formula for the field gradient of the EF wiggler given by Eq. (5) seems quite different from that given by Eq. (3), it can be transformed to a simple expression similar to Eq. (3) as

$$
\frac{\partial B_{y}}{\partial x} \approx 3.5 \frac{B_{T 0}}{\lambda_{W}} \phi,
$$

where $B_{T 0}$ is given by

$$
\begin{aligned}
B_{T 0}= & 2 B_{r} \cdot \exp \left(-\pi g / \lambda_{T}\right) \cdot\left(\frac{\sin (\pi / 2)}{\pi / 2}\right) \\
& \cdot\left\{1-\exp \left(-2 \pi h / \lambda_{T}\right)\right\},
\end{aligned}
$$

which is the peak magnetic field of a wiggler with the period length of $\lambda_{T}$ and $M=2$. Differences between 
Eqs. (3) and (6) are the peak magnetic fields, $B_{0}$ and $B_{T 0}$, and the coefficients, 4 and 3.5. For the experimental model of the EF wiggler with the magnet gap of $30 \mathrm{~mm}, B_{0}=$ $0.432 \mathrm{~T}$ and $B_{T 0}=0.5 \mathrm{~T}$ but the field gradient calculated by Eq. (3), $1.0 \mathrm{~T} / \mathrm{m}$, is close to a value calculated by Eq. (6), $1.02 \mathrm{~T} / \mathrm{m}$. This agreement of the field gradients is accidental because magnet gap dependencies of $B_{0}$ and $B_{T 0}$ are different for the experimental model. When $\lambda_{W}$ is equal to $\lambda_{T}$, the gap dependency of field gradient derived from Eq. (3) and (5) is the same but their absolute values are different by a factor of 1.6. Equation (3) derived with an alternating dipole model may be applicable to a longperiod and small-gap wiggler made of electromagnets with iron poles, in which the magnetic field distribution is rectangular and uniform on the top, but it should not be used for the EF wiggler.

\section{CONCLUSION}

In order to advance development of the EF wiggler, we fabricated the experimental model of the EF wiggler. The magnetic field in the wiggler was precisely measured using Hall probes at four wiggler gaps. It has been experimentally confirmed that a high field gradient of about $1.0 \mathrm{~T} / \mathrm{m}$ is realized, as expected, along the beam axis in the EF wiggler, which is superimposed on the normal wiggler field. We obtained the relation between the magnetic field gradient and the magnetic gap of the EF wiggler. The field gradient exponentially decreases with increasing magnet gap more slowly than the peak magnetic field does for the experimental model, which is favorable in view of the beam focusing in the wiggler. We thus evaluated the performance of the EF wiggler experimentally with the magnetic field measurement. We then derived an analytic formula for the field gradient of the EF wiggler. It is found that the change of the field gradient with the magnet gap can be varied by the magnet width of the EF wiggler.

\section{ACKNOWLEDGMENTS}

The authors would like to thank Mr. T. Kohda and Mr. K. Okihira of NEOMAX Co., Ltd. for their help in the fabrication and the magnetic field measurement of the $\mathrm{EF}$ wiggler. The authors would like to thank Mr. K. Kobayashi and Mr. K. Huruhashi of the Graduate School of Science, Osaka University, for their help in the magnetic field measurement of the EF wiggler at KEK. This research was partly supported by the Joint Development Research at the High Energy Accelerator Research Organization (KEK), 2003-17, 2003, and the Ministry of Education, Science, Sports and Culture, Grant-in-Aid for Exploratory Research, 15654036, 2003 and 2004.

\section{APPENDIX A}

The vertical and the horizontal magnetic fields in the EF wiggler are measured with two Hall probes mounted on a 3-axis linear stage, and the vertical and the horizontal gradients are derived from the measured magnetic fields. If the Hall probes and the linear stage for them have orientation errors, the measured magnetic field has an error in strength and even a small orientation error may result in a large error in the field gradient as well as the field strength for the EF wiggler. The orientation error of a Hall probe results in intrusion of the other components and the orientation error of the linear stage presents the magnetic field at a different point, both of which produce both field errors and gradient errors or either of them.

The orientation error of a Hall probe may be defined as shown schematically in Fig. 14. A Hall probe measures a component of the magnetic field normal to its plane and it is insensitive to its rotation around the normal axis, so that the orientation error may be defined by the polar angle and the azimuthal angle $(\theta, \phi)$ as shown in Fig. 14. The white circle with black outline represents a Hall probe with its normal axis $a_{3}$ ideally aligned to one of the axes defined for the wiggler, $X, Y$, or $Z$, and the gray circle with red outline represents a Hall probe with an orientation error. Two Hall probes are used for measurement of the horizontal and the vertical magnetic fields, and their orientation errors may be specified by $\left(\theta_{x}, \phi_{x}\right)$ and $\left(\theta_{y}, \phi_{y}\right)$, respectively. The orientation errors of the 3-axis linear stage on which the Hall probes are mounted may be defined similarly as shown in Fig. 15. The axis $s_{3}$ denotes the ideal direction of motion and the orientation error is defined by the polar angle $\psi_{3}$ and the azimuthal angle $\zeta_{3}$. Three linear stages comprising the 3-axis linear stage may have independent and different orientation errors, so that they are denoted by $\left(\psi_{x}, \zeta_{x}\right)$, $\left(\psi_{y}, \zeta_{y}\right)$, and $\left(\psi_{z}, \zeta_{z}\right)$. The origin of the coordinate system

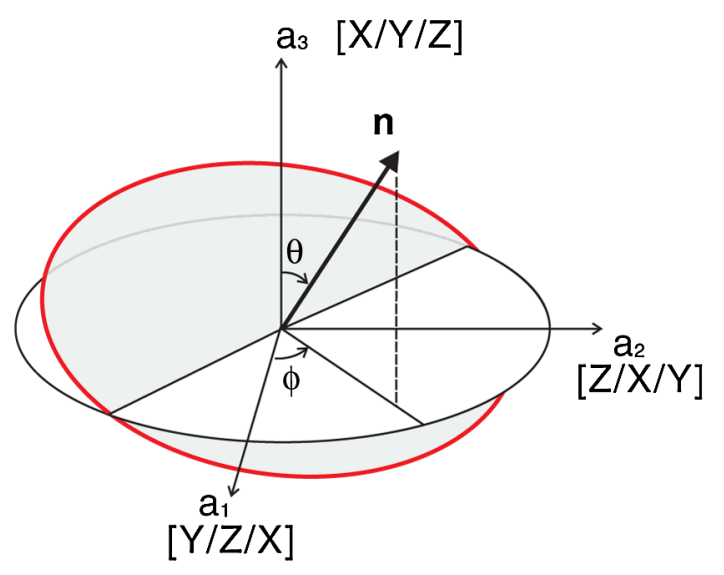

FIG. 14. (Color) Coordinate system for defining the orientation error of a Hall probe. The white circle with black outline shows a Hall probe with no orientation error and $\left(a_{1}, a_{2}, a_{3}\right)$ is the coordinate system attached to it, which may be related to the coordinate system for the wiggler $(X, Y, Z)$. The gray circle with the red outline shows a Hall probe with an orientation error defined by the azimuthal angle $\phi$ and the polar angle $\theta$. The vector $\mathbf{n}$ is a unit vector normal to the Hall probe with the orientation error. 


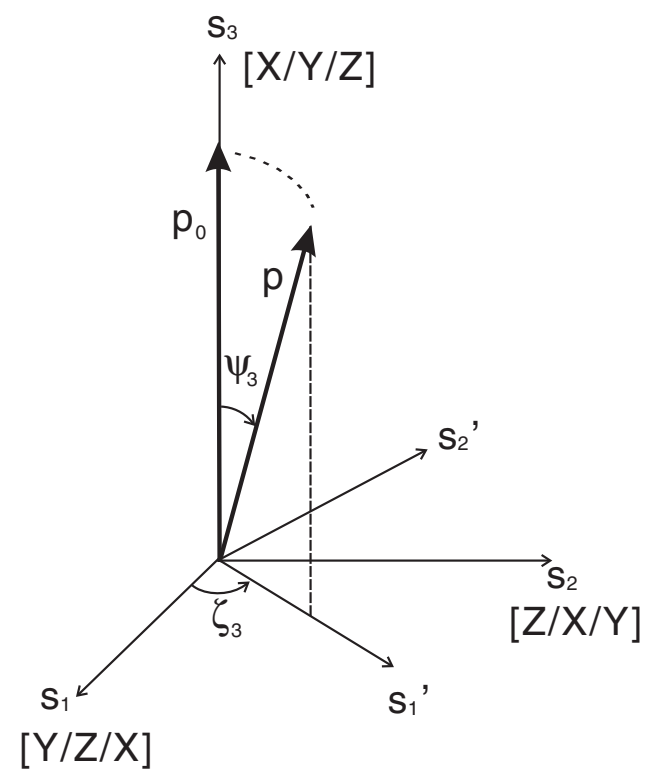

FIG. 15. Coordinate system for defining the orientation error of a linear stage and the position of the Hall probe. The coordinate system $\left(s_{1}, s_{2}, s_{3}\right)$ is defined for a linear stage with no orientation error and $s_{3}$ is its moving axis. The orientation error of the moving axis is defined by the angles $\left(\psi_{3}, \zeta_{3}\right)$. The vectors $\mathbf{p}_{\mathbf{0}}$ and $\mathbf{p}$ show positions of the Hall probe without and with the angular error, respectively.

is calibrated to be the magnetic center of the $\mathrm{EF}$ wiggler and the $\left(s_{1}, s_{2}, s_{3}\right)$ stands for one of the cyclic permutations of $(X, Y, Z)$ defined for the magnetic field measurement.

In order to analyze the orientation errors of the measurement system and to correct the measured magnetic field or field gradient, we need a model magnetic field of the EF wiggler $\mathbf{B}^{\text {model }}$ and it is assumed as follows:

$$
\begin{gathered}
B_{x}^{\text {model }}=G y \quad B_{y}^{\text {model }}=B_{0} \cosh (k y) \cos (k z)+G x \\
B_{z}^{\text {model }}=-B_{0} \sinh (k y) \sin (k z),
\end{gathered}
$$

where $B_{0}$ is the peak magnetic field of the ideal planar wiggler, $k=2 \pi / \lambda_{w}$, and $G$ is the field gradient. The field gradient has a small $z$ dependence with a period of $\lambda_{w} / 2$ determined by the mechanical structure of the EF wiggler.

\section{Magnetic field}

We first consider correction for the measured magnetic field against the orientation errors. The horizontal and the vertical magnetic fields $B_{x}{ }^{\text {mes }}$ and $B_{y}{ }^{\text {mes }}$ measured with the Hall probes with the orientation errors may be given by

$$
\begin{aligned}
B_{x}^{\text {mes }} & =B_{y} \sin \theta_{x} \cos \phi_{x}+B_{z} \sin \theta_{x} \sin \phi_{x}+B_{x} \cos \theta_{x} \\
& \cong B_{x} \pm B_{z} \theta_{x} \\
B_{y}^{\text {mes }} & =B_{z} \sin \theta_{y} \cos \phi_{y}+B_{x} \sin \theta_{y} \sin \phi_{y}+B_{y} \cos \theta_{y} \cong B_{y},
\end{aligned}
$$

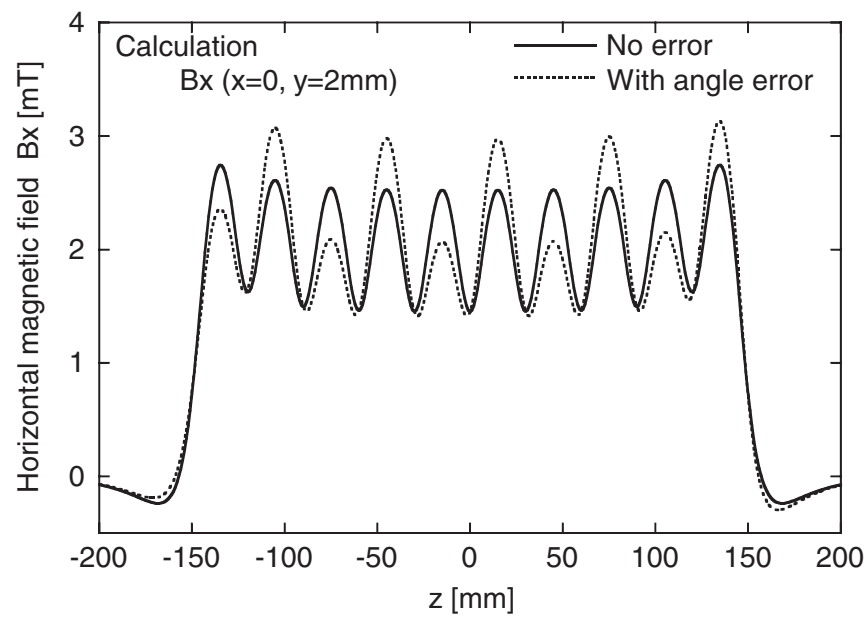

FIG. 16. Horizontal magnetic fields $\left(B_{x}\right)$ at $x=0 \mathrm{~mm}$ and $y=$ $2 \mathrm{~mm}$ calculated along the wiggler axis $z$ with no orientation error (solid lines) and with an orientation error $\left(\theta_{x}=\right.$ $\left.5.0 \mathrm{mrad}, \phi_{x}=-\pi / 2\right)$ of the Hall probe (dashed lines).

where $\mathbf{B}=\left(B_{x}, B_{y}, B_{z}\right)$ is the magnetic field in the $\mathrm{EF}$ wiggler. When the horizontal magnetic field $B_{x}$ is measured, the orientation of the Hall probe is adjusted at a position of the highest $B_{y}$ on the median plane, where $B_{x}$ and $B_{z}$ are zero owing to the structural symmetry, so that intrusion of $B_{y}$ becomes minimum and $B_{x}{ }^{\text {mes }}$ becomes zero. Because this adjustment makes $\phi_{x}= \pm \pi / 2$ but $\theta_{x}$ is indefinite, $B_{z}$ mingles in the measured field $B_{x}{ }^{\text {mes }}$. Figure 16 shows a result of simulated measurement of the horizontal magnetic fields with and without the orientation error. The horizontal magnetic fields of the EF wiggler are calculated off the median plane at $x=0$ and $y=2 \mathrm{~mm}$ along the longitudinal position and an orientation error of the Hall probe $\left(\theta_{x}=5 \mathrm{mrad}, \phi_{x}=\pi / 2\right)$ is assumed. The horizontal magnetic field without the error, which is plotted with the solid line, shows an oscillation with the period $\lambda_{w} / 2$, while the peaks of the magnetic field with the error, plotted with the dotted line, becomes alternatively high and low because of the mixing of the longitudinal magnetic field $B_{z}$, which changes its direction with the period $\lambda_{w}$ as can be seen in Eq. (A2a). The polar angle of the orientation error $\theta_{x}$ may be estimated with Eq. (A2a) and $B_{z}{ }^{\text {model }}$ given in Eq. (A1) so that this alternative variation is diminished.

For measurement of the vertical magnetic field, the orientation error of the Hall probe is corrected at a position on the median plane where the vertical magnetic field is the highest, as in the case of the horizontal magnetic field. Since $B_{x}$ and $B_{z}$ are zero on the median plane and accordingly the first and the second terms of the center of Eq. (A2b) become zero, the polar angle $\theta_{y}$ may be uniquely set to be zero by maximizing the measured magnetic field, so that the vertical magnetic field can be measured without the orientation error. 
The horizontal magnetic fields shown in Fig. 5(b) are those corrected for the orientation error of $\theta_{x}=0.7 \mathrm{mrad}$ and $\phi_{x}=\pi / 2$ estimated by the method. Because it is not possible to estimate the azimuthal angle $\phi_{x}$ uniquely by this method, it is assumed to be $\phi_{x}=\pi / 2$, which gives the minimum value of $\theta_{x}$.

The orientation error of the 3-axis linear stage may affect the measured magnetic field. Let $(a, b, c)$ be the spurious position measured with linear scales attached to the linear stages and $(x, y, z)$ be the actual position, then they are related as

$$
\begin{aligned}
x & =a+\delta x_{x}+\delta x_{y}+\delta x_{z} \\
& =a \cos \psi_{x}+b \sin \psi_{y} \sin \zeta_{y}+c \sin \psi_{z} \cos \zeta_{z} \\
y & =b+\delta y_{x}+\delta y_{y}+\delta y_{z} \\
& =a \sin \psi_{x} \cos \zeta_{x}+b \cos \psi_{y}+c \sin \psi_{z} \sin \zeta_{z} \\
z & =c+\delta z_{x}+\delta z_{y}+\delta z_{z} \\
& =a \sin \psi_{x} \sin \zeta_{x}+b \sin \psi_{y} \cos \zeta_{y}+c \cos \psi_{z} .
\end{aligned}
$$

The horizontal and the vertical magnetic fields are given, respectively, by

$$
\begin{aligned}
B_{x}(x, y, z) \cong & B_{x}^{\operatorname{mes}}(a, b, c)+\frac{\partial B_{x}(a, b, c)}{\partial x}\left[a\left(\cos \psi_{x}-1\right)+b \sin \psi_{y} \sin \phi_{y}+c \sin \psi_{z} \cos \zeta_{z}\right] \\
& +\frac{\partial B_{x}(a, b, c)}{\partial y}\left[a \sin \psi_{x} \cos \zeta_{x}+b\left(\cos \psi_{y}-1\right)+c \sin \psi_{z} \sin \zeta_{z}\right] \\
& +\frac{\partial B_{x}(a, b, c)}{\partial z}\left[a \sin \psi_{x} \sin \zeta_{x}+b \sin \psi_{y} \cos \zeta_{y}+c\left(\cos \psi_{z}-1\right)\right]
\end{aligned}
$$

and

$$
\begin{aligned}
B_{y}(x, y, z) \cong & B_{y}^{\operatorname{mes}}(a, b, c)+\frac{\partial B_{y}(a, b, c)}{\partial x}\left[a\left(\cos \psi_{x}-1\right)+b \sin \psi_{y} \sin \zeta_{y}+c \sin \psi_{z} \cos \zeta_{z}\right] \\
& +\frac{\partial B_{y}(a, b, c)}{\partial y}\left[a \sin \psi_{x} \cos \zeta_{x}+b\left(\cos \psi_{y}-1\right)+c \sin \psi_{z} \sin \zeta_{z}\right] \\
& +\frac{\partial B_{y}(a, b, c)}{\partial z}\left[a \sin \psi_{x} \sin \zeta_{x}+b \sin \psi_{y} \cos \zeta_{y}+c\left(\cos \psi_{z}-1\right)\right] .
\end{aligned}
$$

The magnetic fields are measured along the central axis of the EF wiggler, so that we may set $a=b=0$. If the partial derivatives of the model magnetic field are substituted to Eqs. (A4a) and (A4b), then the magnetic fields are given by

$$
\begin{aligned}
& B_{x}(0,0, z) \cong B_{x}^{\operatorname{mes}}(0,0, c)+G c \psi_{z} \sin \zeta_{z} \\
& B_{y}(0,0, z) \cong B_{y}^{\operatorname{mes}}(0,0, c)+G c \psi_{z} \cos \zeta_{z} .
\end{aligned}
$$

The linear stage for the $z$ direction can be precisely aligned to the EF wiggler with an orientation error of the polar angle $\psi_{z}$ less than $50 \mu \mathrm{rad}$, so that the second terms of the right side in Eqs. (A5a) and (A5b) can be eliminated. Thus, the orientation errors of the 3-axis linear stage do not affect the measured magnetic field under the present experimental conditions.

\section{Field gradient}

The horizontal and the vertical field gradients with the orientation errors of the Hall probes are given by

$$
\begin{aligned}
\frac{\partial B_{x}^{\text {mes }}}{\partial y}= & \frac{\partial B_{y}}{\partial y} \sin \theta_{x} \cos \phi_{x}+\frac{\partial B_{z}}{\partial y} \sin \theta_{x} \sin \phi_{x} \\
& +\frac{\partial B_{x}}{\partial y} \cos \theta_{x} \cong \frac{\partial B_{x}}{\partial y} \pm \frac{\partial B_{z}}{\partial y} \theta_{x} \sin \phi_{x} \\
\frac{\partial B_{y}^{\text {mes }}}{\partial x}= & \frac{\partial B_{z}}{\partial x} \sin \theta_{y} \cos \phi_{y}+\frac{\partial B_{x}}{\partial x} \sin \theta_{y} \sin \phi_{y} \\
& +\frac{\partial B_{y}}{\partial x} \cos \theta_{y} \cong \frac{\partial B_{y}}{\partial x}
\end{aligned}
$$

By the adjustment of the Hall probes in the way described in Appendix A 1, the orientation errors may be reduced to be $\phi_{x}= \pm \pi / 2$ and $\theta_{y}=0$, so that the field gradients are approximately given by the right sides of Eqs. (A6a) and (A6b). There is no orientation error in the vertical field gradient $\left(\partial B_{y} / \partial x\right)$, while the polar angle $\theta_{x}$ remains in the horizontal field gradient $\left(\partial B_{x} / \partial y\right)$. Figure 17 shows results of simulated measurement of the horizontal field gradient with and without an orientation error of the Hall probe $\left(\theta_{x}=5 \mathrm{mrad}, \phi_{x}=-\pi / 2\right)$. The field gradients with and without the orientation error shown in Fig. 17 are exactly similar to the horizontal magnetic fields in Fig. 16. If the partial derivative of the model field is substituted to Eq. (A6a), the horizontal gradient along the central axis is 
given by

$$
\frac{\partial B_{x}^{\text {mes }}(0,0, z)}{\partial y} \cong \frac{\partial B_{x}(0,0, z)}{\partial y} \mp B_{0} k \sin (k z) \theta_{x} \sin \phi_{x} .
$$

The orientation error $\theta_{x}$ may be easily estimated with the horizontal field gradient derived from the measured magnetic field and the model magnetic field in the same way as it is used for the horizontal magnetic field. The orientation errors derived from the horizontal magnetic field and the horizontal gradient should be equal to each other.

The horizontal field gradient shown with the solid circles in Fig. 8(b) is corrected for the orientation error of $\theta_{x}=$ $0.7 \mathrm{mrad}$ and $\phi_{x}=\pi / 2$ estimated by the method. This orientation error of the Hall probe estimated from the horizontal gradient is used for correction of the horizontal magnetic fields shown in Fig. 5(b).

The field gradients produced by the orientation errors of the 3-axis linear stage may be given by

$$
\begin{aligned}
\frac{\partial B_{x}(x, y, z)}{\partial y} \cong & \cos \psi_{y} \frac{\partial B_{x}(x, y, z)}{\partial b} \\
\cong & \frac{\partial B_{x}^{\operatorname{mes}}(a, b, c)}{\partial b}+\frac{\partial B_{x}(a, b, c)}{\partial x} \psi_{y} \sin \zeta_{y} \\
& +\frac{\partial B_{x}(a, b, c)}{\partial z} \psi_{y} \cos \zeta_{y} \cong \frac{\partial B_{x}^{\text {mes }}(a, b, c)}{\partial b}
\end{aligned}
$$

$$
\begin{aligned}
\frac{\partial B_{y}(x, y, z)}{\partial x} \cong & \cos \psi_{x} \frac{\partial B_{y}(x, y, z)}{\partial a} \\
\cong & \frac{\partial B_{y}^{\text {mes }}(a, b, c)}{\partial a}+\frac{\partial B_{y}(a, b, c)}{\partial y} \psi_{x} \cos \zeta_{x} \\
& +\frac{\partial B_{y}(a, b, c)}{\partial z} \psi_{x} \sin \zeta_{x} \cong \frac{\partial B_{y}^{\text {mes }}(a, b, c)}{\partial a} \\
& -B_{0} k \sinh (k b) \cos (k c) \psi_{x} \cos \zeta_{x} \\
& -B_{0} k \cosh (k b) \sin (k c) \psi_{x} \sin \zeta_{x},
\end{aligned}
$$

where the partial derivatives of the model magnetic fields are substituted in Eqs. (A8a) and (A8b). The horizontal field gradient is not affected by the orientation error of the linear stage, while the vertical field gradient is. Figure 18 shows results of simulation for vertical field gradients with and without the orientation error of the linear stage. The vertical field gradient in the EF wiggler is calculated along the central axis at $x=0$ and $y=0 \mathrm{~mm}$ and an orientation error of the Hall probe $\left(\theta_{x}=5 \mathrm{mrad}, \phi_{x}=-\pi / 2\right)$ is assumed. The vertical field gradient with the error varies with the longitudinal position exactly the same as the horizontal field gradient with the orientation error of the Hall probe shown in Fig. 17. The orientation error of the horizontal linear stage mixes $\left(\partial B_{y} / \partial z\right)$ in the vertical field gradient, while the orientation error of the Hall probe for the horizontal direction mixes $\left(\partial B_{z} / \partial y\right)$ in the horizontal

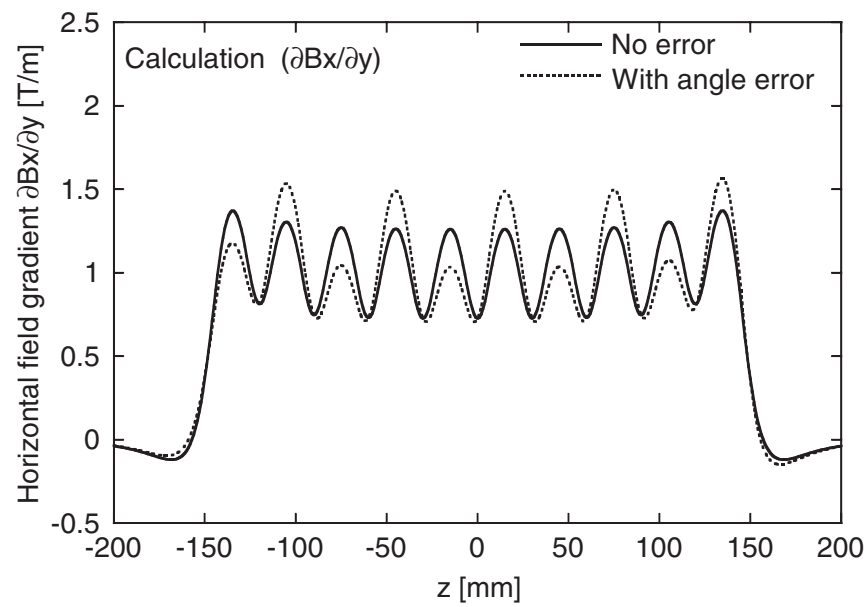

FIG. 17. Horizontal field gradients $\left(\partial B_{x} / \partial y\right)$ at $x=0$ calculated along the wiggler axis $z$ with no orientation error (solid lines) and with an orientation error $\left(\theta_{x}=5.0 \mathrm{mrad}, \phi_{x}=\right.$ $-\pi / 2$ ) of the Hall probe (dashed lines).

field gradient. The polar angle of the orientation error $\psi_{x}$ for $\zeta_{x}= \pm \pi / 2$ may be estimated from the measured horizontal gradient along the central axis of the EF wiggler at $a=b=0$ using

$$
\frac{\partial B_{y}(0,0, z)}{\partial x} \cong \frac{\partial B_{y}^{\text {mes }}(0,0, c)}{\partial a} \mp B_{0} k \sin (k c) \psi_{x}
$$

and $\left(\partial B_{y}{ }^{\text {model }} / \partial z\right)$ or the partial derivative calculated from the measured vertical magnetic field $B_{y}$ so that this alternative variation is diminished. The measured vertical field gradients shown in Figs. 8(a) and 9(a) are corrected against an orientation error of the linear stage of $\psi_{x}=0.9 \mathrm{mrad}$ and $\zeta_{x}=\pi / 2$ so that the symmetry is restored.

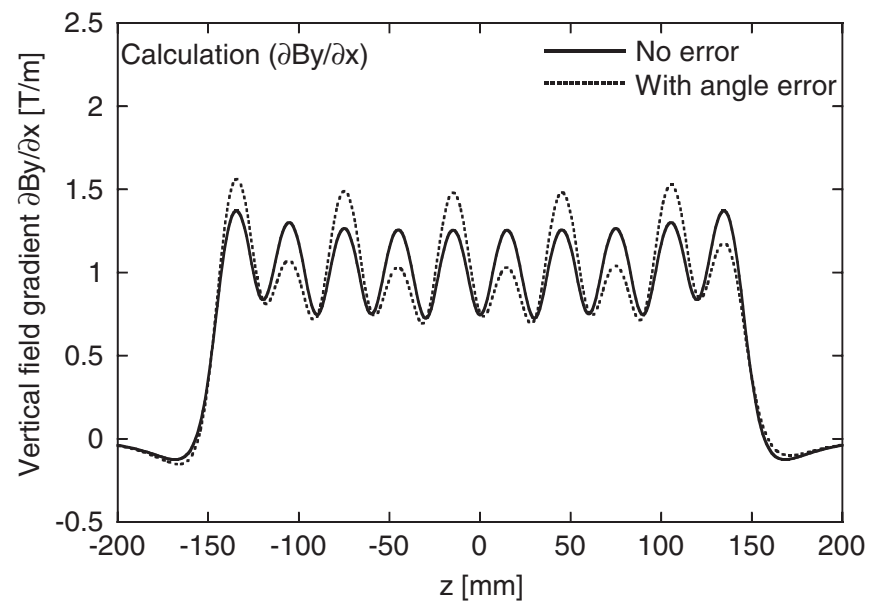

FIG. 18. Vertical field gradients $(\partial B y / \partial x)$ calculated along the wiggler axis with no error (solid line) and with an orientation error of $\psi_{x}=5.0 \mathrm{mrad}$ and $\zeta_{x}=\pi / 2$ in the linear stage for the horizontal direction $x$. 
[1] J. M. J. Madey, J. Appl. Phys. 42, 1906 (1971).

[2] L. R. Elias, W. M. Fairbank, J. M. J. Madey, H. A. Schwettman, and T. I. Smith, Phys. Rev. Lett. 36, 717 (1976).

[3] B. Bonifacio, N. Narducci, and C. Pellegrini, Opt. Commun. 50, 373 (1984).

[4] K.-J. Kim, Phys. Rev. Lett. 57, 1871 (1986).

[5] J. Rossbach, Nucl. Instrum. Methods Phys. Res., Sect. A 475, 13 (2001).

[6] T. Shintake, T. Tanaka, T. Hara, K. Togawa, T. Inagaki, Y. J. Kim, T. Ishikawa, H. Kitamura, H. Baba, H. Matsumoto, S. Takeda, M. Yoshida, and Y. Takasu, Nucl. Instrum. Methods Phys. Res., Sect. A 507, 382 (2003).

[7] LCLS Design Study Report, SLAC-R-521, UC-414, 1998.

[8] E. Trakhtenberg, V. Tcheskidov, I. Vasserman, N. Vinokurov, M. Erdmann, and J. Pfluger, Nucl. Instrum. Methods Phys. Res., Sect. A 543, 42 (2005).

[9] J. Pflüger and M. Tischer, Nucl. Instrum. Methods Phys. Res., Sect. A 483, 388 (2002).

[10] M. Takabe, M. Ogoshi, M. Marushita, H. Sakae, K. Araki, S. Mandai, T. Keishi, and A. Kobayashi, Nucl. Instrum. Methods Phys. Res., Sect. A 331, 736 (1993).

[11] Y. Tsunawaki, N. Ohigashi, K. Mima, T. Akiba, S. Kuruma, K. Imasaki, S. Nakai, and L. R. Elias, Nucl. Instrum. Methods Phys. Res., Sect. A 304, 753 (1991).

[12] M. Shiho, K. Sakamoto, S. Maebara, A. Watanabe, Y. Kishimoto, H. Oda, S. Kawasaki, T. Nagashima, and H. Maeda, Nucl. Instrum. Methods Phys. Res., Sect. A 304, 141 (1991).

[13] Y. Wasa, T. Suzuki, T. Kusaka, K. Inoue, Y. Kawata, A. Kobayashi, and T. Keishi, Nucl. Instrum. Methods Phys. Res., Sect. A 331, 715 (1993).

[14] J. Pflüger and Y.M. Nikitina, Nucl. Instrum. Methods
Phys. Res., Sect. A 381, 554 (1996).

[15] A. A. Varfolomeev and A. H. Hairetdinov, Nucl. Instrum. Methods Phys. Res., Sect. A 341, 462 (1994).

[16] A. A. Varfolomeev, V. V. Gubankov, A. H. Hairetdinov, S. N. Ivanchenkov, A.S. Khlebnikov, N.S. Osmanov, and S. V. Tolmachev, Nucl. Instrum. Methods Phys. Res., Sect. A 358, 70 (1995).

[17] Ross D. Schlueter, Nucl. Instrum. Methods Phys. Res., Sect. A 358, 44 (1995).

[18] R. Carr, M. Cornacchia, P. Emma, H.-D. Nuhn, B. Poling, R. Ruland, E. Johnson, G. Rakowsky, J. Skaritka, S. Lidia, P. Duffy, M. Libkind, P. Frigola, A. Murokh, C. Pellegrini, J. Rosenzweig, and A. Tremaine, Phys. Rev. ST Accel. Beams 4, 122402 (2001).

[19] G. Isoyama, M. Fujimoto, R. Kato, S. Yamamoto, and K. Tsuchiya, Nucl. Instrum. Methods Phys. Res., Sect. A 507, 234 (2003).

[20] S. Kashiwagi, A. Mihara, R. Kato, G. Isoyama, S. Yamamoto, and K. Tsuchiya, Nucl. Instrum. Methods Phys. Res., Sect. A 528, 203 (2004).

[21] R. Kato, T. Okita, R. A. V. Kumar, T. Igo, T. Konishi, M. Kuwahara, M. Fujimoto, S. Mitani, S. Okuda, S. Suemine, and G. Isoyama, Nucl. Instrum. Methods Phys. Res., Sect. A 475, 334 (2001).

[22] R. Kato, R. A. V. Kumar, T. Okita, S. Kondo, T. Igo, T. Konishi, S. Okuda, S. Suemine, and G. Isoyama, Nucl. Instrum. Methods Phys. Res., Sect. A 445, 164 (2000).

[23] H. Wiedemann, Particle Accelerator Physics II (SpringerVerlag, New York, 1995), Chap. 2.

[24] G. Isoyama, Rev. Sci. Instrum. 60, 1826 (1989).

[25] Hitachi Metals, Ltd. (private communication).

[26] G. Brown, K. Halbach, J. Harris, and H. Winick, Nucl. Instrum. Methods 208, 65 (1983). 